\title{
Article
}

\section{Proinflammatory and Hepatic Features Related to Morbidity and Fatal Outcomes in COVID-19 Patients}

\author{
Omar Ramos-Lopez ${ }^{1,+}{ }^{\mathbb{D}}$, Rodrigo San-Cristobal ${ }^{2,+}{ }^{\mathbb{D}}$, Diego Martinez-Urbistondo ${ }^{3}$, Víctor Micó ${ }^{4}$ (D), \\ Gonzalo Colmenarejo ${ }^{5}$ (D), Paula Villares-Fernandez ${ }^{3}$, Lidia Daimiel ${ }^{4}$ (D) and J. Alfredo Martinez ${ }^{2,6,7,8, *}$
}

Citation: Ramos-Lopez, O.;

San-Cristobal, R.;

Martinez-Urbistondo, D.; Micó, V.;

Colmenarejo, G.; Villares-Fernandez,

P.; Daimiel, L.; Martinez, J.A.

Proinflammatory and Hepatic

Features Related to Morbidity and Fatal Outcomes in COVID-19 Patients. J. Clin. Med. 2021, 10, 3112. https:// doi.org/10.3390/jcm10143112

Academic Editors: Yasuhito Tanaka and Robert Flisiak

Received: 19 May 2021

Accepted: 12 July 2021

Published: 15 July 2021

Publisher's Note: MDPI stays neutral with regard to jurisdictional claims in published maps and institutional affiliations.

Copyright: (C) 2021 by the authors Licensee MDPI, Basel, Switzerland. This article is an open access article distributed under the terms and conditions of the Creative Commons Attribution (CC BY) license (https:// creativecommons.org/licenses/by/ $4.0 /)$.
1 Medicine and Psychology School, Autonomous University of Baja California, Tijuana 22390, Mexico; oscar.omar.ramos.lopez@uabc.edu.mx

2 Precision Nutrition and Cardiometabolic Health, IMDEA Food Institute, CEI UAM+CSIC, 28049 Madrid, Spain; rodrigo.sancristobal@imdea.org

3 Hospital Universitario HM Sanchinarro, 28050 Madrid, Spain; dmurbistondo@gmail.com (D.M.-U.); pvillares@hmhospitales.com (P.V.-F.)

4 Nutritional Control of the Epigenome Group, IMDEA Food Institute, CEI UAM+CSIC, 28049 Madrid, Spain; victor.mico@imdea.org (V.M.); lidia.daimiel@imdea.org (L.D.)

5 Biostatistics and Bioinformatics Unit, IMDEA Food Institute, CEI UAM+CSIC, 28049 Madrid, Spain; gonzalo.colmenarejo@imdea.org

6 Department of Nutrition, Food Science, Physiology and Toxicology, Centre for Nutrition Research, University of Navarra, 31009 Pamplona, Spain

7 Navarra Institute for Health Research (IdiSNA), 31009 Pamplona, Spain

8 Spanish Biomedical Research Centre in Physiopathology of Obesity and Nutrition (CIBERobn), 28029 Madrid, Spain

* Correspondence: jalfredo.martinez@imdea.org; Tel.: +34-948-425600

+ Both authors contributed equally to this work.

Abstract: Objective: to screen putative associations between liver markers and proinflammatoryrelated features concerning infectious morbidity and fatal outcomes in COVID-19 patients. Methods: a total of 2094 COVID-19 positive patients from the COVID-DATA-SAFE-LIFES cohort (HM hospitals consortium) were classified according to median values of hepatic, inflammatory, and clinical indicators. Logistic regression models were fitted and ROC cures were generated to explain disease severity and mortality. Results: intensive care unit (ICU) assistance plus death outcomes were associated with liver dysfunction, hyperinflammation, respiratory insufficiency, and higher associated comorbidities. Four models including age, sex, neutrophils, D-dimer, oxygen saturation lower than $92 \%$, C-reactive protein (CRP), Charlson Comorbidity Index (CCI), FIB-4 and interactions with CRP, neutrophils, and CCI explained ICU plus death variance in more than $28 \%$. The predictive values of ROC curves were: FIB-4 (0.7339), AST / ALT ratio (0.7107), CRP (0.7003), CCI index (0.6778), neutrophils (0.6772), and platelets $(0.5618)$ concerning ICU plus death outcomes. Conclusions: the results of this research revealed that liver and proinflammatory features are important determinants of COVID-19 morbidity and fatal outcomes, which could improve the current understanding of the COVID-19 physiopathology as well as to facilitate the clinical management and therapy decision-making of this disease under a personalized medicine scope.

Keywords: liver markers; inflammation; morbidity; mortality; personalized medicine

\section{Introduction}

Coronavirus disease 2019 (COVID-19), caused by the Severe Acute Respiratory Syndrome Coronavirus 2 (SARS-COV-2), has being declared as a pandemic by the World Health Organization (WHO) in March 2020 based on the rises in the daily number of new cases, fast and ample spread, lethality, and the lack of effective antiviral treatments [1]. Since COVID-19 emergence in Wuhan, China, in December 2019, millions of COVID-19 cases have been reported worldwide, with a wide spectrum of respiratory presentations and multisystemic complications [2]. 
The excessive immunological reaction to the virus (known as "cytokine storm") by the host is largely responsible for the respiratory manifestations of COVID-19, encompassing pneumonia and acute respiratory distress syndrome (ARDS); however, in some patients this response may also involve hepatic, gastrointestinal, cardiac, renal, neurological, and hematological affectations [3]. Concerning liver injuries, large-scale case studies indicate that up to $11 \%$ of patients developed liver comorbidities, and more than $50 \%$ of cases reported abnormal levels of transaminases during disease progression, whereas liver dysfunction was more prevalent in severe COVID-19 patients [4]. In such patients, liver damage seems to be directly caused by the viral infection of liver cells, drug toxicity, and immune-mediated inflammation [5]. However, further studies are needed to understand and elucidate the precise causes of liver disease in COVID-19.

Until now, certain clinical, demographic, and phenotypical factors have been reported to be associated with the evolution and severity of COVID-19, encompassing age, sex, ethnicity, underlying medical conditions such as obesity, diabetes, and hypertension, poverty and crowding, pregnancy, and the use of certain medications and genetics [6,7]. Others include elevated levels of proinflammatory cytokines, liver enzymes, coagulation factors, body temperature, and unhealthy lifestyle such as smoking and alcoholic drinks consumption [8]. Nonetheless, there is a constant need for the search for easily accessible, rapid and accurate markers related to the course of COVID-19, which could contribute to improving the individualized clinical management and monitoring of the progression of this infection through an integrative precision medicine approach [9]. The aim of this research was to screen putative associations between available liver markers and proinflammatory-related features concerning infectious morbidity and fatal outcomes in COVID-19 patients.

\section{Methods}

\subsection{Database and Study Variables}

In this retrospective study, data from emergency admission of 2094 COVID-19 positive patients from the COVID-DATA-SAFE-LIFES cohort were analyzed. This cohort contains data on 2226 patients treated for COVID-19 in the HM group hospitals in the first wave of infections (March-May 2020), which has been made available to the international scientific community for study upon appropriate request and approval by a Committee expressly appointed by the hospital consortium (CEIm HM Hospitales Ref No. 20.05.1627-GHM) and under appropriate ethical protocols (Helsinki Declaration).

All data were recorded according to in-hospital protocols, which were harmonized and curated for further analysis in the R software (version 4.0.3). The study variables analyzed in this investigation at baseline comprised age, sex, oxygen saturation, leukocytes, lymphocytes, neutrophils, platelets, basophils, eosinophils, monocytes, C-reactive protein (CRP), D-dimer, fibrinogen, ferritin, procalcitonin, glucose, cholesterol, lactate dehydrogenase (LDH), gamma glutamyl transferase (GGT), aspartate aminotransferase or glutamic oxaloacetic transaminase (AST/GOT), and alanine transaminase or glutamate pyruvate transaminase (ALT/GPT). The following inflammatory-related ratios were calculated: international normalized ratio (INR), AST/ALT ratio (AAR), basophil-to-lymphocyte ratio (BLR), neutrophil-to-lymphocyte ratio (NLR), platelet-to-lymphocyte ratio (PLR), eosinophil-to-basophil ratio (EBR), eosinophil-to-lymphocyte ratio (ELR), and lymphocyteto-monocyte ratio (LMR). Moreover, the Charlson Comorbidity Index (CCI) was computed to express the sum of co-morbidities. As non-invasive methods for predicting liver fibrosis [10], the following scores were calculated:

AST to Platelet Ratio Index (APRI): APRI = [(AST/upper limit of the normal AST range) $\times 100] /$ Platelet Count.

Fibrosis-4 index (FIB-4): FIB-4 = Age $($ years $) \times$ AST $(U / L) /\left[\right.$ platelet $\operatorname{count}\left(10^{9} / \mathrm{L}\right) \times$ $\left.\mathrm{ALT}^{1 / 2}(\mathrm{U} / \mathrm{L})\right]$. 


\subsection{Statistical Analyses}

Quantitative and qualitative variables were expressed as means \pm standard deviations (SD) and as number and percentage, respectively. Chi-square and Student's t-test were applied to analyze differences between qualitative and quantitative variables, as appropriate. Death and ICU were combined and used as main outcomes since these are objective criteria of poor prognosis, as reported elsewhere [11]. Phenotypical and metabolic characteristics of the COVID-19 patients were compared by the median values of hepatic (FIB-4), inflammatory (CRP, neutrophils), and clinical markers (CCI index and oxygen saturation) by Student's $t$-test. Multivariable logistic regression models were fitted to explain disease severity and mortality, with age and sex as covariates. Age was excluded from the CCI index in the models to avoid colinearity. Statistical associations were calculated by univariate logistic regression tests. In addition, area under the receiver operating characteristic (ROC) curves were built to evaluate the predictive values of clinically relevant variables. Statistical analyses were performed in the statistical program Stata 12 (StataCorp LLC, College Station, TX, USA; www.stata.com (accessed on 2 May 2021)) and IBM SPSS 20 (IBM Inc., Armonk, NY, USA). Statistical significance was set at $p$ value lower than 0.05 , with bilateral test.

\section{Results}

The clinical and phenotypical characteristics of COVID-19 patients based on respiratory insufficiency, comorbidity, or need of intensive care plus mortality risk are reported (Table 1). On average, individuals with oxygen saturation lower than $92 \%$, CCI index equal or higher than 3 , and those who underwent ICU or who died were male, older and presented higher levels of leukocytes, neutrophils, CRP, D-dimer, LDH, FIB-4 as well as elevated ratios of AAR, basophil-to-lymphocyte, neutrophil-to-lymphocyte, plateletto-lymphocyte, and lymphocyte-to-monocyte than their counterparts. Conversely, no differences between groups were observed for basophils, procalcitonin, glucose, cholesterol, and GPT measurements.

Similar features were found when compared the median values of inflammatory (CRP and neutrophils) and liver (FIB-4) markers in COVID-19 patients (Table 2).

Logistic regression models using relevant biochemical and clinical variables to predict ICU plus death outcome were constructed, with age and sex as covariates. Interestingly, four models were statistically significant $(p<0.001)$ and explained ICU plus death variance in more than $28 \%$ (Table $3 \mathrm{a}-\mathrm{d}$ ). The models included age, sex, neutrophils, D-dimer, oxygen saturation $<92 \%$, CRP, CCI index, FIB- 4 , and the following interactions: CCI index $\times$ CRP (Table 3a); FIB-4 * CCI index (Table 3b); FIB- 4 * CRP (Table 3c); and FIB- 4 * neutrophils (Table $3 \mathrm{~d}$ ), respectively. The four interactions were statistically significant in the corresponding models. 
Table 1. Clinical and phenotypical characteristics of COVID-19 patients based on respiratory insufficiency (oxygen saturation), comorbidities (CCI), and ICU plus death outcomes.

\begin{tabular}{|c|c|c|c|c|c|c|c|c|c|}
\hline \multirow[b]{2}{*}{ Variable } & \multicolumn{2}{|c|}{ Oxygen Saturation $\left(\mathrm{SO}_{2} \%\right)$} & \multirow[b]{2}{*}{$p$} & \multicolumn{2}{|c|}{ CCI } & \multicolumn{4}{|c|}{ ICU and Death } \\
\hline & $\begin{array}{c}\geq 92 \% \\
(n=494)\end{array}$ & $\begin{array}{c}<92 \% \\
(n=1314)\end{array}$ & & $\begin{array}{c}<3 \\
(n=781) \\
\end{array}$ & $\begin{aligned} & \geq 3 \\
(n & =994)\end{aligned}$ & $p$ & $\begin{array}{c}\text { No ICU + No Death } \\
(n=1645)\end{array}$ & $\begin{array}{c}\text { ICU + Death } \\
(n=449)\end{array}$ & $p$ \\
\hline Age & $66.7 \pm 16.7$ & $72.8 \pm 13.4$ & $<0.001$ & $52.2 \pm 11.7$ & $76.3 \pm 10.7$ & $<0.001$ & $64.4 \pm 16.0$ & $76.2 \pm 14.1$ & $<0.001$ \\
\hline CCI & $2.90 \pm 2.38$ & $4.01 \pm 2.41$ & $<0.001$ & $1.00 \pm 0.83$ & $4.87 \pm 1.93$ & $<0.001$ & $2.70 \pm 2.21$ & $5.15 \pm 2.55$ & $<0.001$ \\
\hline Oxygen saturation $\left(\mathrm{SO}_{2} \%\right)$ & $95.4 \pm 2.0$ & $84.4 \pm 9.1$ & $<0.001$ & $94.0 \pm 4.3$ & $91.0 \pm 8.3$ & $<0.001$ & $93.6 \pm 5.1$ & $87.2 \pm 10.8$ & $<0.001$ \\
\hline Leukocytes $\left(\times 10^{9} / \mathrm{L}\right)$ & $7.13 \pm 3.77$ & $9.09 \pm 6.04$ & $<0.001$ & $6.89 \pm 3.15$ & $8.00 \pm 5.32$ & $<0.001$ & $7.14 \pm 3.63$ & $9.62 \pm 6.65$ & $<0.001$ \\
\hline Lymphocytes $\left(\times 10^{9} / \mathrm{L}\right)$ & $1.27 \pm 1.51$ & $1.01 \pm 0.79$ & $<0.001$ & $1.23 \pm 0.60$ & $1.15 \pm 1.61$ & 0.172 & $1.24 \pm 1.30$ & $1.04 \pm 1.27$ & 0.007 \\
\hline Platelets $\left(\times 10^{9} / \mathrm{L}\right)$ & $219.4 \pm 93.5$ & $231.2 \pm 98.4$ & 0.023 & $225.6 \pm 91.4$ & $218.1 \pm 94.9$ & 0.107 & $227.4 \pm 95.7$ & $210.6 \pm 100.1$ & 0.004 \\
\hline Basophils $\left(\times 10^{9} / \mathrm{L}\right)$ & $0.022 \pm 0.021$ & $0.022 \pm 0.021$ & 0.526 & $0.021 \pm 0.022$ & $0.022 \pm 0.020$ & 0.475 & $0.021 \pm 0.020$ & $0.022 \pm 0.021$ & 0.400 \\
\hline Eosinophils $\left(\times 10^{9} / \mathrm{L}\right)$ & $0.05 \pm 0.26$ & $0.02 \pm 0.06$ & 0.032 & $0.04 \pm 0.08$ & $0.04 \pm 0.29$ & 0.527 & $0.05 \pm 0.23$ & $0.02 \pm 0.05$ & 0.036 \\
\hline Monocytes $\left(\times 10^{9} / \mathrm{L}\right)$ & $0.56 \pm 0.37$ & $0.68 \pm 3.30$ & 0.254 & $0.52 \pm 0.29$ & $0.63 \pm 2.44$ & 0.241 & $0.54 \pm 0.30$ & $0.75 \pm 3.67$ & 0.044 \\
\hline $\mathrm{CRP}(\mathrm{mg} / \mathrm{L})$ & $79.3 \pm 83.2$ & $158.4 \pm 113.8$ & $<0.001$ & $83.4 \pm 85.0$ & $114.7 \pm 107.5$ & $<0.001$ & $87.3 \pm 87.1$ & $161.3 \pm 124.8$ & $<0.001$ \\
\hline D-dimer $(\mu \mathrm{g} / \mathrm{mL})$ & $1.97 \pm 6.72$ & $3.33 \pm 8.80$ & 0.003 & $1.08 \pm 2.56$ & $3.19 \pm 10.04$ & $<0.001$ & $1.75 \pm 5.5$ & $4.92 \pm 13.21$ & $<0.001$ \\
\hline Fibrinogen (mg/dL) & $638.7 \pm 175.3$ & $772.9 \pm 348.4$ & 0.035 & $696.1 \pm 209.6$ & $683.4 \pm 193.8$ & 0.763 & $646.8 \pm 176.3$ & $776.4 \pm 307.0$ & 0.002 \\
\hline Ferritin $(\mathrm{ng} / \mathrm{mL})$ & $867 \pm 701$ & $2870 \pm 2931$ & $<0.001$ & $2030 \pm 3427$ & $1313 \pm 1345$ & 0.226 & $1363 \pm 2213$ & $1812 \pm 1484$ & 0.392 \\
\hline Procalcitonin (ng/mL) & $0.08 \pm 0.03$ & $1.02 \pm 2.01$ & 0.157 & $0.33 \pm 0.89$ & $1.00 \pm 1.76$ & 0.142 & $0.41 \pm 0.89$ & $0.89 \pm 1.64$ & 0.209 \\
\hline Glucose (mg/dL) & $208.0 \pm 97.6$ & $177.0 \pm 30.7$ & 0.545 & $128.5 \pm 34.1$ & $216.8 \pm 102.5$ & 0.117 & $200.2 \pm 105.7$ & $169.0 \pm 60.0$ & 0.545 \\
\hline $\mathrm{LDH}(\mathrm{U} / \mathrm{L})$ & $546.1 \pm 257.7$ & $786.6 \pm 411.2$ & $<0.001$ & $562.3 \pm 256.0$ & $646.9 \pm 406.9$ & $<0.001$ & $563.5 \pm 249.3$ & $816.1 \pm 570.5$ & $<0.001$ \\
\hline GGT (U/L) & $74.2 \pm 91.4$ & $157.0 \pm 345.4$ & 0.025 & $74.5 \pm 93.3$ & $74.0 \pm 170.0$ & 0.978 & $74.4 \pm 90.1$ & $89.2 \pm 211.7$ & 0.420 \\
\hline GOT (U/L) & $43.1 \pm 110.9$ & $58.7 \pm 90.9$ & 0.012 & $44.3 \pm 32.9$ & $50.9 \pm 141.6$ & 0.265 & $42.4 \pm 32.8$ & $66.6 \pm 208.2$ & $<0.001$ \\
\hline GPT (U/L) & $37.5 \pm 82.5$ & $46.3 \pm 66.0$ & 0.054 & $45.7 \pm 46.1$ & $38.5 \pm 100.2$ & 0.108 & $39.5 \pm 40.6$ & $45.9 \pm 144.1$ & 0.178 \\
\hline INR ratio & $1.43 \pm 1.25$ & $1.43 \pm 1.18$ & 0.995 & $1.25 \pm 0.49$ & $1.55 \pm 1.75$ & $<0.001$ & $1.34 \pm 0.99$ & $1.69 \pm 2.10$ & $<0.001$ \\
\hline AAR ratio & $1.39 \pm 0.72$ & $1.56 \pm 0.74$ & $<0.001$ & $1.17 \pm 0.51$ & $1.58 \pm 0.76$ & $<0.001$ & $1.32 \pm 0.66$ & $1.74 \pm 0.76$ & $<0.001$ \\
\hline APRI score & $0.59 \pm 1.34$ & $0.72 \pm 0.77$ & 0.073 & $0.57 \pm 0.54$ & $0.68 \pm 1.55$ & 0.105 & $0.55 \pm 0.54$ & $0.89 \pm 2.24$ & $<0.001$ \\
\hline FIB-4 score & $2.66 \pm 3.00$ & $3.31 \pm 2.75$ & $<0.001$ & $1.86 \pm 1.20$ & $3.51 \pm 3.66$ & $<0.001$ & $2.43 \pm 2.22$ & $4.24 \pm 4.48$ & $<0.001$ \\
\hline BLR & $0.02 \pm 0.02$ & $0.03 \pm 0.06$ & $<0.001$ & $0.02 \pm 0.02$ & $0.02 \pm 0.05$ & $<0.001$ & $0.02 \pm 0.03$ & $0.03 \pm 0.04$ & $<0.001$ \\
\hline NLR & $5.57 \pm 5.27$ & $10.78 \pm 18.67$ & $<0.001$ & $5.18 \pm 4.48$ & $8.24 \pm 14.32$ & $<0.001$ & $5.92 \pm 10.64$ & $11.43 \pm 10.76$ & $<0.001$ \\
\hline PLR & $220.1 \pm 139.5$ & $303.8 \pm 315.0$ & $<0.001$ & $213.7 \pm 126.5$ & $264.6 \pm 253.9$ & $<0.001$ & $230.8 \pm 198.1$ & $296.5 \pm 225.0$ & $<0.001$ \\
\hline EBR & $1.87 \pm 4.31$ & $1.06 \pm 2.35$ & $<0.001$ & $1.61 \pm 2.85$ & $1.66 \pm 4.58$ & 0.800 & $1.89 \pm 4.19$ & $1.01 \pm 2.47$ & $<0.001$ \\
\hline ELR & $0.03 \pm 0.10$ & $0.02 \pm 0.05$ & 0.054 & $0.02 \pm 0.04$ & $0.03 \pm 0.11$ & 0.024 & $0.03 \pm 0.09$ & $0.02 \pm 0.06$ & 0.119 \\
\hline LMR & $2.71 \pm 2.07$ & $2.33 \pm 1.56$ & $<0.001$ & $2.82 \pm 1.74$ & $2.40 \pm 1.70$ & $<0.001$ & $2.66 \pm 1.83$ & $2.43 \pm 2.23$ & 0.040 \\
\hline
\end{tabular}

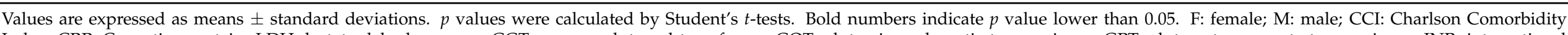

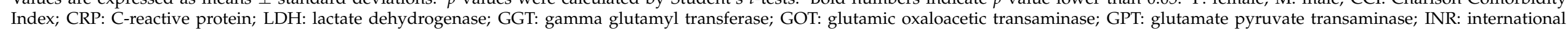

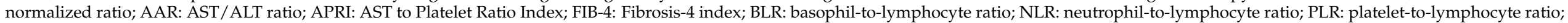
EBR: eosinophil-to-basophil ratio; ELR: eosinophil-to-lymphocyte ratio; LMR: lymphocyte-to-monocyte ratio. 
Table 2. Clinical and phenotypical characteristics of COVID-19 patients based on median values of inflammatory and liver features.

\begin{tabular}{|c|c|c|c|c|c|c|c|c|c|}
\hline \multirow[b]{2}{*}{ Variable } & \multicolumn{2}{|c|}{ CRP } & \multirow[b]{2}{*}{$p$} & \multicolumn{2}{|c|}{ Neutrophils } & \multicolumn{4}{|c|}{ FIB-4 } \\
\hline & $\begin{array}{c}<73.67 \\
(n=933)\end{array}$ & $\begin{array}{c}\geq 73.67 \\
(n=934)\end{array}$ & & $\begin{array}{c}<4.89 \\
(n=976)\end{array}$ & $\begin{array}{c}\geq 4.89 \\
(n=982)\end{array}$ & $p$ & $\begin{array}{c}<2.17 \\
(n=810)\end{array}$ & $\begin{array}{c}\geq 2.17 \\
(n=818)\end{array}$ & $p$ \\
\hline Age & $65.1 \pm 17.2$ & $69.9 \pm 14.5$ & $<0.001$ & $65.2 \pm 16.0$ & $69.8 \pm 15.7$ & $<0.001$ & $61.0 \pm 15.8$ & $74.3 \pm 13.0$ & $<0.001$ \\
\hline $\operatorname{Sex}(\mathrm{F} / \mathrm{M})$ & $425 / 508$ & $315 / 618$ & $<0.001$ & $416 / 560$ & $363 / 618$ & 0.003 & $350 / 460$ & $281 / 537$ & $<0.001$ \\
\hline $\mathrm{CCI}$ & $2.86 \pm 2.42$ & $3.42 \pm 2.33$ & $<0.001$ & $2.87 \pm 2.34$ & $3.43 \pm 2.46$ & $<0.001$ & $2.36 \pm 2.28$ & $4.01 \pm 2.29$ & $<0.001$ \\
\hline Oxygen saturation $\left(\mathrm{SO}_{2} \%\right)$ & $94.4 \pm 4.0$ & $89.8 \pm 4.4$ & $<0.001$ & $93.9 \pm 4.5$ & $90.3 \pm 8.4$ & $<0.001$ & $93.1 \pm 6.0$ & $90.8 \pm 8.0$ & $<0.001$ \\
\hline Leukocytes $\left(\times 10^{9} / \mathrm{L}\right)$ & $6.58 \pm 3.46$ & $8.66 \pm 5.11$ & $<0.001$ & $5.07 \pm 2.98$ & $10.20 \pm 4.27$ & $<0.001$ & $8.15 \pm 3.99$ & $7.18 \pm 4.99$ & $<0.001$ \\
\hline Lymphocytes $\left(\times 10^{9} / \mathrm{L}\right)$ & $1.37 \pm 1.61$ & $0.99 \pm 0.66$ & $<0.001$ & $1.18 \pm 0.72$ & $1.19 \pm 1.62$ & 0.172 & $1.28 \pm 0.81$ & $1.07 \pm 1.70$ & 0.002 \\
\hline Neutrophils $\left(\times 10^{9} / \mathrm{L}\right)$ & $4.57 \pm 2.71$ & $7.01 \pm 4.18$ & $<0.001$ & $3.31 \pm 0.95$ & $8.30 \pm 3.80$ & $<0.001$ & $6.17 \pm 3.76$ & $5.51 \pm 3.55$ & $<0.001$ \\
\hline Platelets $\left(\times 10^{9} / \mathrm{L}\right)$ & $217.9 \pm 94.9$ & $231.7 \pm 98.5$ & 0.002 & $195.1 \pm 80.1$ & $254.2 \pm 104.4$ & 0.107 & $275.1 \pm 102.8$ & $177.0 \pm 59.9$ & $<0.001$ \\
\hline Basophils $\left(\times 10^{9} / \mathrm{L}\right)$ & $0.021 \pm 0.022$ & $0.022 \pm 0.020$ & 0.527 & $0.017 \pm 0.018$ & $0.024 \pm 0.021$ & 0.475 & $0.025 \pm 0.021$ & $0.017 \pm 0.016$ & $<0.001$ \\
\hline Eosinophils $\left(\times 10^{9} / \mathrm{L}\right)$ & $0.06 \pm 0.28$ & $0.03 \pm 0.05$ & 0.001 & $0.03 \pm 0.07$ & $0.04 \pm 0.27$ & 0.527 & $0.06 \pm 0.30$ & $0.02 \pm 0.05$ & $<0.001$ \\
\hline Monocytes $\left(\times 10^{9} / \mathrm{L}\right)$ & $0.56 \pm 0.31$ & $0.60 \pm 2.34$ & 0.548 & $0.52 \pm 2.31$ & $0.63 \pm 0.41$ & 0.241 & $0.60 \pm 0.32$ & $0.56 \pm 2.50$ & 0.692 \\
\hline $\mathrm{CRP}(\mathrm{mg} / \mathrm{L})$ & $31.2 \pm 21.4$ & $173.6 \pm 97.4$ & $<0.001$ & $62.9 \pm 61.1$ & $142.3 \pm 115.1$ & $<0.001$ & $95.8 \pm 103.3$ & $113.4 \pm 98.6$ & $<0.001$ \\
\hline D-dimer $(\mu \mathrm{g} / \mathrm{mL})$ & $1.66 \pm 4.82$ & $3.04 \pm 9.60$ & 0.001 & $1.37 \pm 4.45$ & $3.35 \pm 9.79$ & $<0.001$ & $2.00 \pm 5.59$ & $2.76 \pm 8.95$ & 0.065 \\
\hline Fibrinogen (mg/dL) & $553.4 \pm 122.4$ & $812.3 \pm 240.8$ & $<0.001$ & $627.9 \pm 187.0$ & $725.7 \pm 255.1$ & 0.763 & $687.2 \pm 254.6$ & $692.3 \pm 221.7$ & 0.903 \\
\hline Ferritin $(\mathrm{ng} / \mathrm{mL})$ & $724 \pm 632$ & $2011 \pm 2566$ & $<0.001$ & $1046 \pm 1474$ & $1791 \pm 2437$ & 0.226 & $1414 \pm 2243$ & $1474 \pm 1872$ & 0.883 \\
\hline Cholesterol (mg/dL) & $162.5 \pm 23.3$ & $125.2 \pm 28.8$ & 0.997 & $127.5 \pm 72.8$ & $137.9 \pm 99.4$ & 0.971 & $137.6 \pm 33.6$ & $134.5 \pm 16.3$ & 0.980 \\
\hline $\mathrm{LDH}(\mathrm{U} / \mathrm{L})$ & $521.2 \pm 311.0$ & $714.8 \pm 362.8$ & $<0.001$ & $554.4 \pm 299.9$ & $682.8 \pm 389.4$ & $<0.001$ & $536.4 \pm 218.7$ & $712.2 \pm 445.2$ & $<0.001$ \\
\hline GGT (U/L) & $49.9 \pm 48.7$ & $101.9 \pm 173.5$ & $<0.001$ & $56.6 \pm 59.4$ & $97.5 \pm 177.5$ & 0.978 & $81.5 \pm 102.5$ & $69.1 \pm 157.6$ & 0.404 \\
\hline GOT (U/L) & $38.8 \pm 30.2$ & $55.7 \pm 134.3$ & $<0.001$ & $41.1 \pm 29.5$ & $53.7 \pm 135.5$ & 0.265 & $34.3 \pm 23.6$ & $61.2 \pm 137.2$ & $<0.001$ \\
\hline GPT (U/L) & $36.1 \pm 40.7$ & $44.8 \pm 96.5$ & 0.020 & $34.5 \pm 33.0$ & $46.4 \pm 99.4$ & 0.108 & $38.0 \pm 42.4$ & $43.2 \pm 96.9$ & 0.159 \\
\hline INR ratio & $1.34 \pm 1.04$ & $1.48 \pm 1.44$ & 0.050 & $1.34 \pm 0.97$ & $1.50 \pm 1.56$ & $<0.001$ & $1.36 \pm 1.34$ & $1.47 \pm 1.31$ & 0.144 \\
\hline AAR ratio & $1.40 \pm 1.32$ & $1.50 \pm 0.67$ & 0.054 & $1.45 \pm 0.70$ & $1.44 \pm 1.27$ & $<0.001$ & $1.14 \pm 0.51$ & $1.76 \pm 1.30$ & $<0.001$ \\
\hline APRI score & $0.54 \pm 0.53$ & $0.70 \pm 1.50$ & 0.003 & $0.63 \pm 0.65$ & $0.61 \pm 1.47$ & 0.105 & $0.34 \pm 0.22$ & $0.92 \pm 1.55$ & $<0.001$ \\
\hline FIB-4 score & $2.52 \pm 2.24$ & $3.12 \pm 3.29$ & $<0.001$ & $3.01 \pm 2.66$ & $2.67 \pm 3.15$ & $<0.001$ & $1.37 \pm 0.48$ & $4.30 \pm 3.54$ & $<0.001$ \\
\hline Bas/Lym ratio & $0.02 \pm 0.03$ & $0.03 \pm 0.04$ & $<0.001$ & $0.01 \pm 0.02$ & $0.02 \pm 0.04$ & $<0.001$ & $0.02 \pm 0.04$ & $0.02 \pm 0.03$ & 0.027 \\
\hline $\mathrm{Neu} / \mathrm{Lym}$ ratio & $4.52 \pm 5.45$ & $9.64 \pm 14.02$ & $<0.001$ & $3.54 \pm 2.62$ & $10.56 \pm 14.18$ & $<0.001$ & $6.97 \pm 14.29$ & $7.52 \pm 7.77$ & 0.337 \\
\hline Plat/Lym ratio & $199.8 \pm 141.9$ & $295.8 \pm 255.8$ & $<0.001$ & $199.1 \pm 128.4$ & $293.6 \pm 258.3$ & $<0.001$ & $274.3 \pm 264.9$ & $231.4 \pm 165.1$ & $<0.001$ \\
\hline Eos/Bas ratio & $2.21 \pm 4.86$ & $1.20 \pm 2.34$ & $<0.001$ & $1.88 \pm 3.29$ & $1.54 \pm 4.24$ & 0.800 & $2.13 \pm 4.69$ & $1.24 \pm 2.90$ & $<0.001$ \\
\hline Eos/Lym ratio & $0.04 \pm 0.11$ & $0.03 \pm 0.05$ & 0.010 & $0.02 \pm 0.05$ & $0.03 \pm 0.10$ & 0.024 & $0.041 \pm 0.114$ & $0.020 \pm 0.051$ & $<0.001$ \\
\hline Lym/Mon ratio & $2.82 \pm 1.76$ & $2.44 \pm 2.04$ & $<0.001$ & $3.04 \pm 1.78$ & $2.18 \pm 1.93$ & $<0.001$ & $2.60 \pm 1.84$ & $2.65 \pm 2.07$ & 0.557 \\
\hline
\end{tabular}

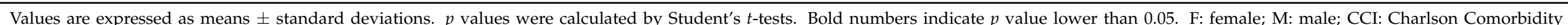

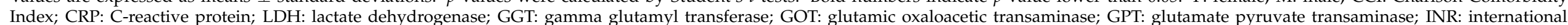

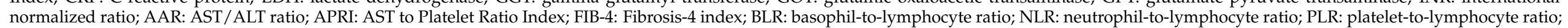
EBR: eosinophil-to-basophil ratio; ELR: eosinophil-to-lymphocyte ratio; LMR: lymphocyte-to-monocyte ratio. 
Table 3. (a) Multiple logistic regression model using clinical, inflammatory and liver markers as important predictors of mortality plus ICU in COVID-19 patients: interaction between CCI and CRP. (b) Multiple logistic regression model using clinical, inflammatory and liver markers as important predictors of mortality plus ICU in COVID-19 patients: interaction between FIB-4 and CCI. (c) Multiple logistic regression model using clinical, inflammatory and liver markers as important predictors of mortality plus ICU in COVID-19 patients: interaction between FIB-4 and CRP. (d) Multiple logistic regression model using clinical, inflammatory and liver markers as important predictors of mortality plus ICU in COVID-19 patients: interaction between FIB-4 and NEU.

\begin{tabular}{|c|c|c|}
\hline \multicolumn{3}{|c|}{ (a) } \\
\hline Variable & $\beta$ Coefficients (CI 95\%) & $p$ \\
\hline Age (years) & $0.0653(0.0464,0.0842)$ & $<0.001$ \\
\hline Sex (Female) & $-0.5413(-1.0090,-0.0735)$ & 0.023 \\
\hline Neutrophils $\left(\times 10^{9} / \mathrm{L}\right)$ & $0.0972(0.0370,0.1574)$ & 0.002 \\
\hline D-dimer $(\mu \mathrm{g} / \mathrm{mL})$ & $0.0218(-0.0037,0.0472)$ & 0.093 \\
\hline Oxygen saturation $\left(\mathrm{SO}_{2}<92 \%\right)$ & $0.6359(0.1909,1.0809)$ & 0.005 \\
\hline FIB- 4 & $0.2080(0.1046,0.3113)$ & $<0.001$ \\
\hline $\mathrm{CCI} * \mathrm{CRP}$ & $0.0013(0.0007,0.0018)$ & $<0.001$ \\
\hline$R^{2}$ & 0.3093 & $<0.001$ \\
\hline \multicolumn{3}{|c|}{ (b) } \\
\hline Variable & $\beta$ coefficients (CI 95\%) & $p$ \\
\hline Age (years) & $0.0776(0.0598,0.0955)$ & $<0.001$ \\
\hline Sex (Female) & $-0.6635(-1.1213,-0.2056)$ & 0.005 \\
\hline Neutrophils $\left(\times 10^{9} / \mathrm{L}\right)$ & $0.0595(-0.0026,0.1216)$ & 0.060 \\
\hline D-dimer $(\mu \mathrm{g} / \mathrm{mL})$ & $0.0249(-0.0009,0.0507)$ & 0.059 \\
\hline Oxygen saturation $\left(\mathrm{SO}_{2}<92 \%\right)$ & $0.6374(0.1976,1.0772)$ & 0.005 \\
\hline $\mathrm{CRP}(\mathrm{mg} / \mathrm{L})$ & $0.0039(0.0017,0.0061)$ & $<0.001$ \\
\hline FIB- 4 * CCI (no age) & $0.0307(0.0137,0.0477)$ & $<0.001$ \\
\hline $\mathrm{R}^{2}$ & 0.2838 & $<0.001$ \\
\hline \multicolumn{3}{|c|}{ (c) } \\
\hline Age (years) & $0.0655(0.0471,0.0839)$ & $<0.001$ \\
\hline Sex (Female) & $-0.5982(-1.0728,-0.1236)$ & 0.013 \\
\hline Neutrophils $\left(\times 10^{9} / \mathrm{L}\right)$ & $0.0763(0.0205,0.1321)$ & 0.007 \\
\hline D-dimer $(\mu \mathrm{g} / \mathrm{mL})$ & $0.0227(-0.0027,0.0482)$ & 0.080 \\
\hline Oxygen saturation $\left(\mathrm{SO}_{2}<92 \%\right)$ & $0.5183(0.0658,0.9709)$ & 0.025 \\
\hline CCI (no age) & $0.2094(0.0962,0.3226)$ & $<0.001$ \\
\hline FIB $-4 *$ CRP & $0.0014(0.0009,0.0020)$ & $<0.001$ \\
\hline $\mathrm{R}^{2}$ & 0.3134 & $<0.001$ \\
\hline \multicolumn{3}{|c|}{ (d) } \\
\hline Age (years) & $0.0601(0.0408,0.0793)$ & $<0.001$ \\
\hline Sex (Female) & $-0.5279(-1.0003,-0.0555)$ & 0.028 \\
\hline CRP (mg/L) & $0.0032(0.0012,0.0053)$ & 0.002 \\
\hline D-dimer $(\mu \mathrm{g} / \mathrm{mL})$ & $0.0225(-0.0027,0.0477)$ & 0.080 \\
\hline Oxygen saturation $\left(\mathrm{SO}_{2}<92 \%\right)$ & $0.5081(0.0519,0.9642)$ & 0.029 \\
\hline CCI (no age) & $0.2202(0.1077,0.3327)$ & $<0.001$ \\
\hline FIB- 4 * NEU & $0.0383(0.0210,0.0556)$ & $<0.001$ \\
\hline $\mathrm{R}^{2}$ & 0.3151 & $<0.001$ \\
\hline
\end{tabular}

Bold numbers indicate $p<0.05$.

The empirical frequencies and odds ratios (OR) of ICU plus death by the cutoffs (median) values of CRP, CCI index, FIB-4, neutrophils, platelets, and AAR ratio are depicted (Figure 1a-f). Compared to patients who did not enter to ICU and did not die, higher risks of ICU plus death were found when CRP levels were equal or higher than $73.67 \mathrm{mg} / \mathrm{L}$ $(\mathrm{OR}=3.475, p<0.001$, Figure 1a); $\mathrm{CCI}$ index equal or higher than $3(\mathrm{OR}=8.040, p<0.001$, Figure 1b); FIB-4 score equal or higher than 2.17 (OR $=3.590, p<0.001$, Figure 1c); neutrophils equal or higher than $4.89 \times 10^{9} / \mathrm{L}(\mathrm{OR}=2.539, p<0.001$, Figure $1 \mathrm{~d})$; and AAR 
ratio equal or higher than 1.29 ( $\mathrm{OR}=3.320, p<0.001$, Figure $1 \mathrm{f})$. Instead, platelets equal or higher than $205 \times 10^{9} / \mathrm{L}$ protected for ICU pus death $(\mathrm{OR}=0.723, p=0.013$, Figure $1 \mathrm{e})$.

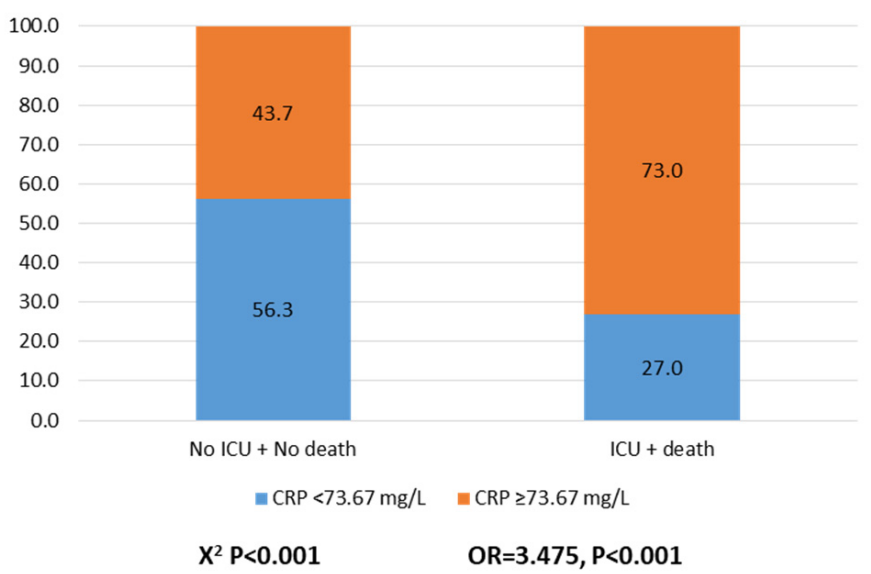

(a) Frequencies of deaths plus ICU by CRP cutoffs

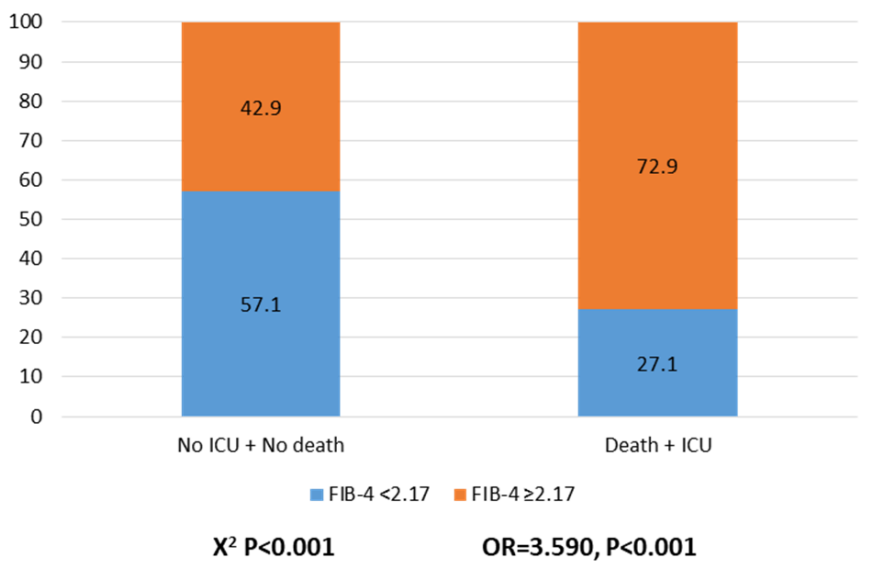

(c) Frequencies of deaths plus ICU by FIB-4 cutoffs

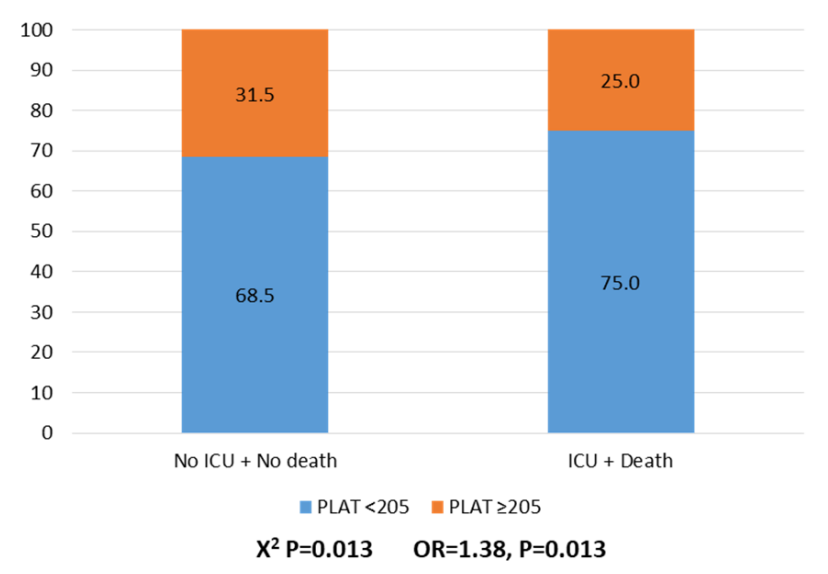

(e) Frequencies of deaths plus ICU by platelets cutoffs

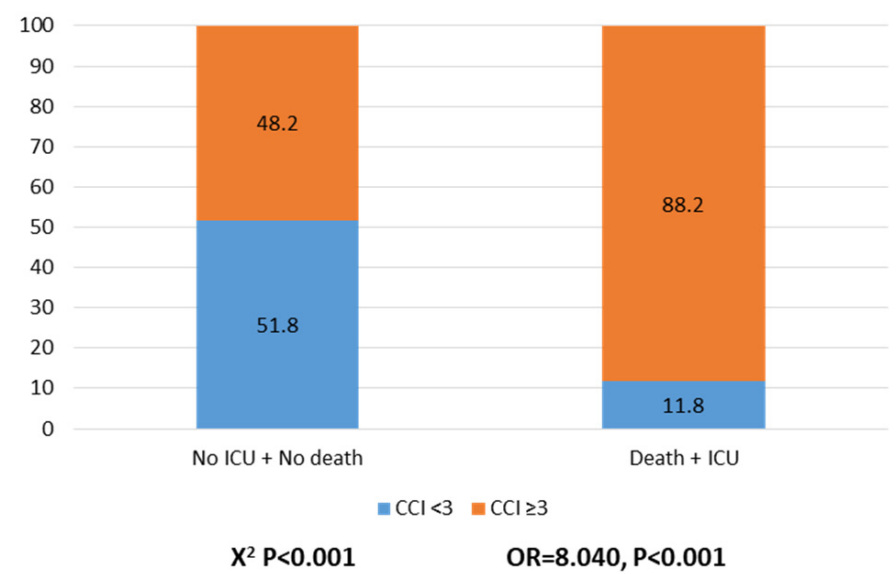

(b) Frequencies of deaths plus ICU by CCI cutoffs

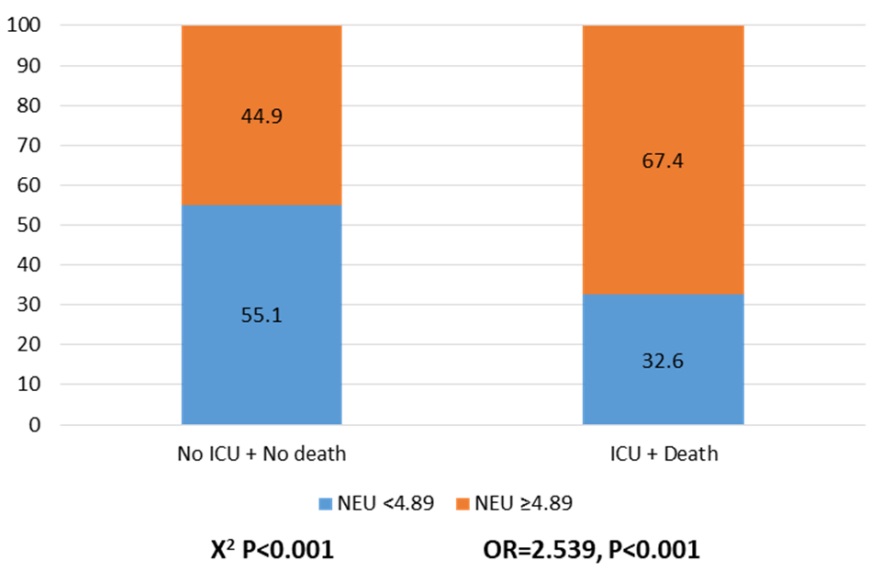

(d) Frequencies of deaths plus IU by NEU cutoffs

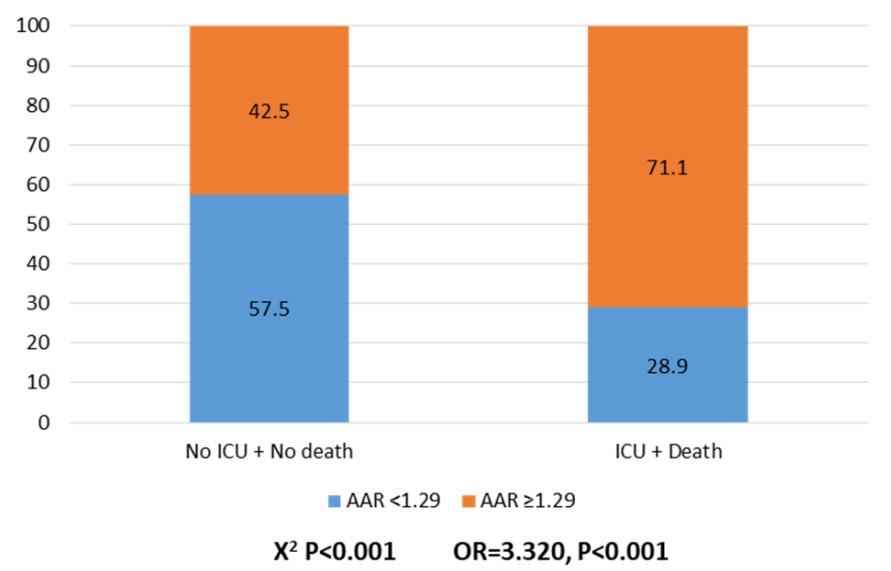

(f) Frequencies of deaths plus ICU by AAR cutoffs

Figure 1. Frequencies and odds ratios (OR) of ICU plus death by the cutoffs (median) values of CRP, CCI index, FIB-4, neutrophils, platelets, and AAR ratio.

ROC curves were constructed to estimate and compare the predictive value of liver and proinflammatory markers concerning ICU plus death (Figure 2). The best predictor was FIB-4 (0.7339), followed by AAR (0.7107), CRP (0.7003), CCI index (0.6778), neutrophils (0.6772), and platelets $(0.5618)$, all of them statistically significant $(p<0.001)$. 


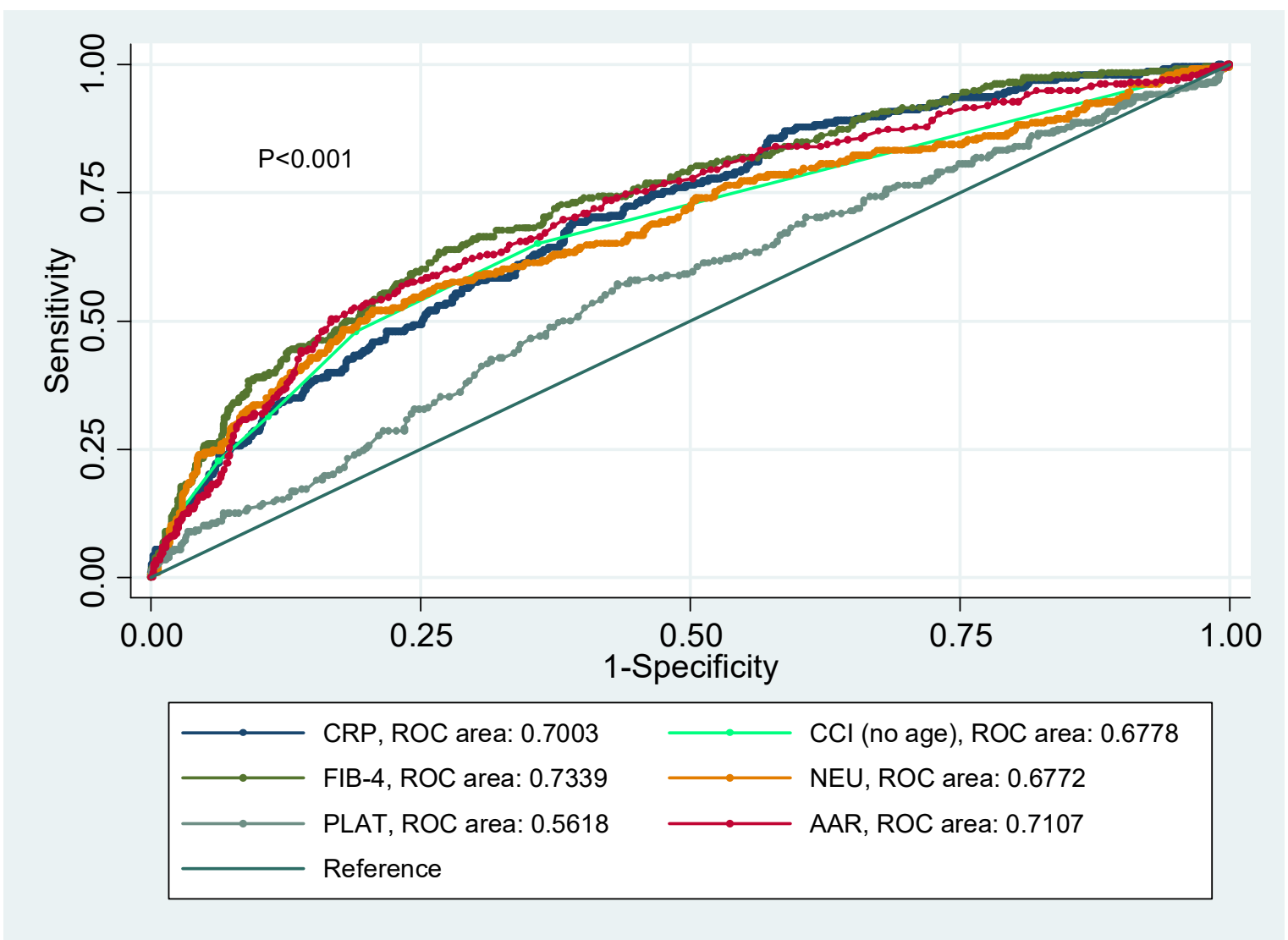

Figure 2. ROC curves showing the predictive value of CCI (no age), CRP, NEU, FIB-4, PLAT, and AAR concerning COVID-19 outcomes (ICU plus Death).

\section{Discussion}

As a result of the increased availability of data and collaborations between researchers, efforts have been made for the evaluation of laboratory tests and other phenotypical information as biomarkers related to COVID-19 disease severity [9]. This study should be considered a proof of concept, where biochemical and clinical variables significantly explained morbid and fatal outcomes in COVID-19 patients, including neutrophils, CRP, oxygen saturation $<92 \%$, FIB-4, D-dimer and CCI index, which evidence the involvement of predominately liver and proinflammatory features in the evolution of this disease. These findings may enable early categorization of infected patients based on the risk of death or intensive care assistance, thus facilitating a more precise clinical management as well as the optimization of health resources and medical personnel [7].

In agreement with our results, neutrophils have been highlighted as essential effector cells in COVID-19 physiopathology through the stimulation of a hyperinflammation state in the lungs by enhanced degranulation of primary granules and the secretion of proinflammatory cytokines as well as the induction of oxidative stress via reactive oxygen species release [12]. In this context, bioinformatic analyses revealed that neutrophil activation is one of the most stimulated biological processes in the SARS-CoV infection [13]. Moreover, it has been reported the association of NLR with critical illness in COVID-19 patients [14].

Likewise, some investigations have confirmed the utility of CRP as prognostic factor in COVID-19 since it serves as an early marker of infection, inflammation, and tissue damage [15]. For example, CRP levels were independent discriminators of severe/critical illness on admission and a good predictor of adverse outcome in COVID-19 patients [16]. In hospitalized patients, median CRP values $(206 \mathrm{mg} / \mathrm{L})$ were significantly higher in the patients who died compared to those who survived, and increased linearly during the first week of hospitalization, which supports the utility of daily CRP monitoring in risk 
prognostication [17]. Accordingly, it has been documented that the risk of developing severe events in COVID-19 patients is increased by about $5 \%$ for every one-unit increase in CRP levels [18]. Interestingly, elevated levels of CRP $(76.51 \mathrm{mg} / \mathrm{L})$ correlated with lower oxygen saturation $(<90 \%)$, indicating a relationship of these markers and a complementary utility in the prognosis of COVID-19 disease [19]. Indeed, oxygen saturation levels below $92 \%$ significantly contributed to predict ICU plus death in this sample. This hallmark is in agreement with the criteria for diagnosis of COVID-19-associated pneumonia and disease severity [20], as postulated in the guidelines of the World Health Organization for the Clinical Management of COVID-19 (https: / / apps.who.int/iris/handle/10665/332196 (accessed on 2 May 2021)). Certainly, $92 \%$ is under the current target oxygen saturation range (92-96\%) for patients with COVID-19 recommended by the National Institutes of Health (https:/ / www.covid19treatmentguidelines.nih.gov/critical-care/oxygenation-andventilation/ (accessed on 2 May 2021)).

Another important finding of this study was the interplay of FIB-4 in COVID-19 disease severity by interacting with proinflammatory and comorbid features. Thus, two statistical interactions were found concerning FIB- 4 and inflammatory markers, where a higher FIB-4 score combined with increased levels of neutrophils and CRP were associated with more instances of ICU plus death (data not shown). These results suggest that an elevated FIB-4 score exacerbates the progression of the inflammatory process, and also suggests an organ-specific influence of inflammation as a prognostic marker. Besides, a significant interaction between CCI and FIB-4 in relation to death plus ICU was found in this research (data not shown), which suggest that when FIB-4 is low, the CCI dominates the entry to ICU admission and the risk of death; however, when FIB-4 is high (above 20), a preservative effect is found. This finding may be explained by the fact that the set of comorbidities (measured by CCI) has a greater influence on the outcomes of patients with COVID-19 than only liver fibrosis (measured by FIB-4). FIB-4 is not only an accurate marker of liver fibrosis, but it is also related to coagulation and oxidative stress since it takes into account age and the serum levels of transaminases (ALT and AST) and platelets, all of which have been consistently identified as potential risk factors of severe cases with COVID19 in a recent meta-analysis [21]. Furthermore, elevation of this FIB-4 (equal or higher than 2.67) was associated with poor clinical outcomes in middle-aged patients with COVID19, including required mechanical ventilation and ICU admission [22]. Moreover, FIB-4 was also related with increased risk of mortality in hospitalized patients with COVID-19 as well as with lower survival [23,24]. In addition, FIB-4 positively correlated to SARSCoV-2 viral load and the levels of inflammatory cytokines [25]. Besides FIB-4, AAR was another liver marker also associated (equal or higher than 1.29) with an increased risk of ICU plus death in this research. Similarly, a retrospective study reported that AAR higher than 1 highly correlated with liver injury in conjunction with other proinflammatory variables [26]. Despite more investigation in this fled is necessary, these results evidence the involvement of liver damage in the evolution of COVID-19 and highlight the importance of evaluate liver status in the clinical setting. Although the role of liver disease in COVID-19 remains unclear, it has been hypothesized that liver injury is associated with innate immune dysfunction, which could enhance susceptibility to an acute proinflammatory response (cytokine storm) leading to severe outcomes in patients with COVID-19 by exacerbating the hyperinflammatory state $[27,28]$. Of note, although the presence of previous liver disease might artifact our findings, the low prevalence in the population (only 53 patients with liver disease) might reduce the confounding effect of this issue. In fact, no significant differences in the performance of the statistical models were found when patients with liver disease were removed.

In relation to the association of abnormal coagulation parameters with poor outcomes in COVID-19 patients, a meta-analysis evidenced that patients with a composite clinical end point, defined as all-cause mortality, ICU admission or ARDS, had elevated levels of D-dimer (standard mean difference of $1.67 \mu \mathrm{g} / \mathrm{mL}$ ) than their counterparts [29]. In fact, 
results from another meta-analysis of 13 cohort studies revealed that severe COVID-19 infection was related to D-dimer higher than $0.5 \mu \mathrm{g} / \mathrm{mL}$ on admission [30].

Regarding comorbidity, in this study, the CCI index was included in the predictive models of ICU plus death mainly as an adjustment variable. The CCI has been commonly used in clinical research as a correction factor in a set of prognostic models due to proven consistency, validity, and reliability as supported by the results of several studies. In COVID-19, multivariate regression analysis showed that CCI was a prognostic factor for COVID-19-related mortality in patients hospitalized for pneumonia [31]. Additionally, CCI score above 0 was associated with an increased risk of severe outcome and death after controlled for age and sex [32]. In a meta-analysis, a 16\% higher risk of mortality was attributed by each per point increase of CCI score [33].

On the one hand, the strengths of this investigation include a large sample screened and the use of robust statistical approaches for data depuration and the comparative predictive analyses. In this context, on the most important findings of this research is the integration of different predictors of COVID-19 outcomes including liver and proinflammatory features as well as the screening of potential interactions among these factors, which suggest that the prognostic value of these markers depends upon the behavior of concomitant variables influencing COVID-19 disease and that there is a mutual influence concerning the result. On the other hand, the fact that the population analyzed in this study has mainly European ancestry, the findings of this study could not be applied to groups with other ethnicity and exposed to diverse environmental factors. For instance, in Latin America, variables such as the high rates of obesity, the adoption of hepatopathogenic diets, and a sedentary lifestyle could exacerbate liver damage and a hyperinflammatory state in COVID-19 [34]. Moreover, the exploration of other variables influencing liver health and the immune response including the gut microbiota, genetic background, epigenetic signatures, metabolomic profiles, and interactions with specific lifestyle factors could be part of the scenario [35]. Additionally, although hyperinflammation worse COVID-19 infection, caution must be taken concerning the interpretation of the results since there can be wide fluctuations in levels of inflammatory markers during the time frame from admission to collection of labs.

In conclusion, the results of this research suggest that liver and proinflammatory features are important determinants of COVID-19 morbid and fatal outcomes. This information could contribute to improve the current comprehension of the COVID-19 physiopathology and the clinical management and therapy decision-making of this disease under a precision medicine approach [36]. Current results evidence that the hepatic responses may have a role in prognosis, treatment, and understanding of COVID-19.

Author Contributions: Conceptualization, O.R.-L. and J.A.M.; formal analysis, R.S.-C., G.C., V.M., L.D., D.M.-U. and P.V.-F.; investigation, O.R.-L., J.A.M., R.S.-C., G.C., V.M., L.D., D.M.-U., and P.V.-F.; data curation, R.S.-C. and V.M.; original draft preparation, O.R.-L., J.A.M.; supervision, D.M.-U. and P.V.-F. All authors have read and agreed to the published version of the manuscript.

Funding: R.S.-C. acknowledges financial support from the Juan de la Cierva Program Training Grants of the Spanish State Research Agency of the Spanish Ministerio de Ciencia e Innovación y Ministerio de Universidades (FJC2018-038168-I).

Institutional Review Board Statement: The study was conducted according to the guidelines of the Declaration of Helsinki, and approved by the Ethics Committee of the HM hospitals consortium (CEIm HM Hospitales Ref No. 20.05.1627-GHM).

Informed Consent Statement: Informed consent was obtained from all subjects involved in the study.

Data Availability Statement: The data presented in this study are available on request from the corresponding author.

Acknowledgments: Authors thank HM hospitals for access to the COVID-DATA-SAFE-LIFES database. 
Conflicts of Interest: The authors declare that they have no conflict of interest concerning this investigation.

\section{References}

1. Tay, M.Z.; Poh, C.M.; Rénia, L.; MacAry, P.A.; Ng, L.F.P. The trinity of COVID-19: Immunity, inflammation and intervention. Nat. Rev. Immunol. 2020, 20, 363-374. [CrossRef]

2. Huang, C.; Wang, Y.; Li, X.; Ren, L.; Zhao, J.; Hu, Y.; Zhang, L.; Fan, G.; Xu, J.; Gu, X.; et al. Clinical features of patients infected with 2019 novel coronavirus in Wuhan, China. Lancet 2020, 395, 497-506. [CrossRef]

3. Lai, C.C.; Ko, W.C.; Lee, P.I.; Jean, S.S.; Hsueh, P.R. Extra-respiratory manifestations of COVID-19. Int. J. Antimicrob. Agents 2020, 56, 106024. [CrossRef]

4. Zhang, C.; Shi, L.; Wang, F.S. Liver injury in COVID-19: Management and challenges. Lancet Gastroenterol. Hepatol. 2020, 5, 428-430. [CrossRef]

5. Cha, M.H.; Regueiro, M.; Sandhu, D.S. Gastrointestinal and hepatic manifestations of COVID-19: A comprehensive review. World J. Gastroenterol. 2020, 26, 2323-2332. [CrossRef]

6. Rod, J.E.; Oviedo-Trespalacios, O.; Cortes-Ramirez, J. A brief-review of the risk factors for covid-19 severity. Rev. Saude Publica 2020, 54, 60. [CrossRef]

7. Ramos-Lopez, O.; Daimiel, L.; Ramírez de Molina, A.; Martínez-Urbistondo, D.; Vargas, J.A.; Martínez, J.A. Exploring Host Genetic Polymorphisms Involved in SARS-CoV Infection Outcomes: Implications for Personalized Medicine in COVID-19. Int. J. Genom. 2020, 2020, 6901217. [CrossRef] [PubMed]

8. Martinez-Urbistondo, M.; Mora-Vargas, A.; Expósito-Palomo, E.; Castejón, R.; Citores, M.J.; Rosado, S.; de Mendoza, C.; Baños, I.; Fernández-Cruz, A.; Daimiel, L.; et al. Inflammatory-Related Clinical and Metabolic Outcomes in COVID-19 Patients. Mediat. Inflamm. 2020, 2020, 2914275. [CrossRef] [PubMed]

9. Moradian, N.; Ochs, H.D.; Sedikies, C.; Hamblin, M.R.; Camargo, C.A., Jr.; Martinez, J.A.; Biamonte, J.D.; Abdollahi, M.; Torres, P.J.; Nieto, J.J.; et al. The urgent need for integrated science to fight COVID-19 pandemic and beyond. J. Transl. Med. 2020, 18, 205. [CrossRef] [PubMed]

10. Martinez-Urbistondo, D.; Suarez Del Villar, R.; Argemí, J.; Daimiel, L.; Ramos-López, O.; San-Cristobal, R.; Villares, P.; Martinez, J.A. Antioxidant Lifestyle, Co-Morbidities and Quality of Life Empowerment Concerning Liver Fibrosis. Antioxidants 2020, 9 , 1125. [CrossRef] [PubMed]

11. Armstrong, R.A.; Kane, A.D.; Cook, T.M. Outcomes from intensive care in patients with COVID-19: A systematic review and meta-analysis of observational studies. Anaesthesia 2020, 75, 1340-1349. [CrossRef] [PubMed]

12. Cavalcante-Silva, L.H.A.; Carvalho, D.C.M.; Lima, É.A.; Galvão, J.G.F.M.; da Silva, J.S.F.; Sales-Neto, J.M.; Rodrigues-Mascarenhas, S. Neutrophils and COVID-19: The road so far. Int. Immunopharmacol. 2020, 90, 107233. [CrossRef]

13. Hemmat, N.; Derakhshani, A.; Bannazadeh Baghi, H.; Silvestris, N.; Baradaran, B.; De Summa, S. Neutrophils, Crucial, or Harmful Immune Cells Involved in Coronavirus Infection: A Bioinformatics Study. Front. Genet. 2020, 11, 641. [CrossRef] [PubMed]

14. Liu, J.; Liu, Y.; Xiang, P.; Pu, L.; Xiong, H.; Li, C.; Zhang, M.; Tan, J.; Xu, Y.; Song, R.; et al. Neutrophil-to-lymphocyte ratio predicts critical illness patients with 2019 coronavirus disease in the early stage. J. Transl. Med. 2020, 18, 206. [CrossRef] [PubMed]

15. Ali, N. Elevated level of C-reactive protein may be an early marker to predict risk for severity of COVID-19. J. Med. Virol. 2020, 92, 2409-2411. [CrossRef] [PubMed]

16. Luo, X.; Zhou, W.; Yan, X.; Guo, T.; Wang, B.; Xia, H.; Ye, L.; Xiong, J.; Jiang, Z.; Liu, Y.; et al. Prognostic Value of C-Reactive Protein in Patients with Coronavirus 2019. Clin. Infect. Dis. 2020, 71, 2174-2179. [CrossRef]

17. Sharifpour, M.; Rangaraju, S.; Liu, M.; Alabyad, D.; Nahab, F.B.; Creel-Bulos, C.M.; Jabaley, C.S.; Emory COVID-19 Quality \& Clinical Research Collaborative. C-Reactive protein as a prognostic indicator in hospitalized patients with COVID-19. PLoS ONE 2020, 15, e0242400. [CrossRef]

18. Wang, G.; Wu, C.; Zhang, Q.; Wu, F.; Yu, B.; Lv, J.; Li, Y.; Li, T.; Zhang, S.; Wu, C.; et al. C-Reactive Protein Level May Predict the Risk of COVID-19 Aggravation. Open Forum Infect. Dis. 2020, 7, ofaa153. [CrossRef]

19. Xie, J.; Covassin, N.; Fan, Z.; Singh, P.; Gao, W.; Li, G.; Kara, T.; Somers, V.K. Association Between Hypoxemia and Mortality in Patients With COVID-19. Mayo Clin. Proc. 2020, 95, 1138-1147. [CrossRef]

20. Shenoy, N.; Luchtel, R.; Gulani, P. Considerations for target oxygen saturation in COVID-19 patients: Are we under-shooting? BMC Med. 2020, 18, 260. [CrossRef] [PubMed]

21. Ou, M.; Zhu, J.; Ji, P.; Li, H.; Zhong, Z.; Li, B.; Pang, J.; Zhang, J.; Zheng, X. Risk factors of severe cases with COVID-19: A meta-analysis. Epidemiol. Infect. 2020, 148, e175. [CrossRef] [PubMed]

22. Ibáñez-Samaniego, L.; Bighelli, F.; Usón, C.; Caravaca, C.; Carrillo, C.F.; Romero, M.; Barreales, M.; Perelló, C.; Madejón, A.; Marcos, A.C.; et al. Elevation of Liver Fibrosis Index FIB-4 Is Associated With Poor Clinical Outcomes in Patients With COVID-19. J. Infect. Dis. 2020, 222, 726-733. [CrossRef] [PubMed]

23. Sterling, R.K.; Oakes, T.; Gal, T.S.; Stevens, M.P.; deWit, M.; Sanyal, A.J. The Fibrosis-4 Index Is Associated With Need for Mechanical Ventilation and 30-Day Mortality in Patients Admitted With Coronavirus Disease 2019. J. Infect. Dis. 2020, 222, 1794-1797. [CrossRef] 
24. Park, J.G.; Kang, M.K.; Lee, Y.R.; Song, J.E.; Kim, N.Y.; Kweon, Y.O.; Tak, W.Y.; Jang, S.Y.; Lee, C.; Kim, B.S.; et al. DaeguGyeongbuk Liver Study Group (DGLSG). Fibrosis-4 index as a predictor for mortality in hospitalised patients with COVID-19: A retrospective multicentre cohort study. BMJ Open 2020, 10, e041989. [CrossRef]

25. Li, Y.; Regan, J.; Fajnzylber, J.; Coxen, K.; Corry, H.; Wong, C.; Rosenthal, A.; Atyeo, C.; Fischinger, S.; Gillespie, E.; et al. Liver Fibrosis Index FIB-4 Is Associated With Mortality in COVID-19. Hepatol. Commun. 2021, 5, 434-445. [CrossRef]

26. Chen, F.; Chen, W.; Chen, J.; Xu, D.; Xie, W.; Wang, X.; Xie, Y. Clinical features and risk factors of COVID-19-associated liver injury and function: A retrospective analysis of 830 cases. Ann. Hepatol. 2020, 21, 100267. [CrossRef]

27. Xiang, F.; Sun, J.; Chen, P.-H.; Han, P.; Zheng, H.; Cai, S.; Kirk, G.D. Early Elevation of Fibrosis-4 Liver Fibrosis Score Is Associated With Adverse Outcomes Among Patients With Coronavirus Disease 2019. Clin. Infect. Dis. 2020. [CrossRef]

28. Calapod, O.; Marin, A.; Onisai, M.; Tribus, L.; Pop, C.; Fierbinteanu-Braticevici, C. The Impact of Increased Fib-4 Score in Patients with Type II Diabetes Mellitus on COVID-19 Disease Prognosis. Medicina 2021, 57, 434. [CrossRef]

29. Bansal, A.; Singh, A.D.; Jain, V.; Aggarwal, M.; Gupta, S.; Padappayil, R.P.; Nadeem, M.; Joshi, S.; Mian, A.; Greathouse, T.; et al. The association of D-dimers with mortality, intensive care unit admission or acute respiratory distress syndrome in patients hospitalized with coronavirus disease 2019 (COVID-19): A systematic review and meta-analysis. Heart Lung 2021, 50, 9-12. [CrossRef]

30. Yu, H.H.; Qin, C.; Chen, M.; Wang, W.; Tian, D.S. D-dimer level is associated with the severity of COVID-19. Thromb. Res. 2020, 195, 219-225. [CrossRef] [PubMed]

31. Varol, Y.; Hakoglu, B.; Kadri Cirak, A.; Polat, G.; Komurcuoglu, B.; Akkol, B.; Atasoy, C.; Bayramic, E.; Balci, G.; Ataman, S.; et al. COVID Study Group. The impact of charlson comorbidity index on mortality from SARS-CoV-2 virus infection and A novel COVID-19 mortality index: CoLACD. Int. J. Clin. Pract. 2021, 75, e13858. [CrossRef]

32. Christensen, D.M.; Strange, J.E.; Gislason, G.; Torp-Pedersen, C.; Gerds, T.; Fosbøl, E.; Phelps, M. Charlson Comorbidity Index Score and Risk of Severe Outcome and Death in Danish COVID-19 Patients. J. Gen. Intern. Med. 2020, 35, 2801-2803. [CrossRef]

33. Tuty Kuswardhani, R.A.; Henrina, J.; Pranata, R.; Anthonius Lim, M.; Lawrensia, S.; Suastika, K. Charlson comorbidity index and a composite of poor outcomes in COVID-19 patients: A systematic review and meta-analysis. Diabetes Metab. Syndr. 2020, 14, 2103-2109. [CrossRef] [PubMed]

34. Buicu, A.L.; Cernea, S.; Benedek, I.; Buicu, C.F.; Benedek, T. Systemic Inflammation and COVID-19 Mortality in Patients with Major Noncommunicable Diseases: Chronic Coronary Syndromes, Diabetes and Obesity. J. Clin. Med. 2021, 10, 1545. [CrossRef] [PubMed]

35. Cuevas-Sierra, A.; Ramos-Lopez, O.; Riezu-Boj, J.I.; Milagro, F.I.; Martinez, J.A. Diet, Gut Microbiota, and Obesity: Links with Host Genetics and Epigenetics and Potential Applications. Adv. Nutr. 2019, 10, S17-S30. [CrossRef] [PubMed]

36. Omersel, J.; Karas Kuželički, N. Vaccinomics and Adversomics in the Era of Precision Medicine: A Review Based on HBV, MMR, HPV, and COVID-19 Vaccines. J. Clin. Med. 2020, 9, 3561. [CrossRef] [PubMed] 